\title{
A Residual Frequency Offset Compensation Scheme for OFDM System via SAGE Algorithm
}

\author{
Jong-Ho Lee, and Seong-Cheol Kim \\ School of Electrical Engineering and Computer Science \\ Seoul National University \\ Seoul, Korea \\ \{dowar, sckim\}@maxwell.snu.ac.kr
}

\begin{abstract}
In this paper, we propose an iterative detection scheme in the presence of residual frequency offset (RFO) for orthogonal frequency-division multiplexing (OFDM) system using the space-alternating generalized expectation-maximization (SAGE) algorithm. In the proposed algorithm, the expectation step intends to remove the inter-carrier interferences (ICI) due to RFO in the received signals. Then, the maximization step is utilized to estimate the required parameters (i.e. RFO, data symbols and channel state information) using the ICI canceled signals. Simulation results present that the proposed algorithm shows almost ideal performance as long as the normalized RFO value is within 0.2 .
\end{abstract}

Keywords- RFO, ICI, SAGE, OFDM.

\section{INTRODUCTION}

Orthogonal frequency-division multiplexing (OFDM) is an attractive technique to support high-rate data transmission over severely dispersed multipath fading channels. However, it is known to be very sensitive to the carrier frequency synchronization and channel estimation errors [1].

Carrier frequency offset induced by the mismatches of local oscillators in the transmitter and receiver causes the intercarrier interferences (ICI), which may result in significant performance degradation. Several carrier frequency synchronization schemes for OFDM systems are reported in the literatures [2]-[6]. However, the residual frequency offset (RFO) due to imperfect synchronization still generates the ICI and degrades the transmission performance [7]. In order to estimate the RFO, [8] and [9] use the sub-carriers that are not actually modulated, considering the orthogonality between subcarriers.

In this paper, we employ the space-alternating generalized expectation-maximization (SAGE) algorithm [10] to overcome the ICI due to RFO. In the proposed scheme, the expectation step intends to remove the ICI induced by RFO in the received signals and the maximization step is utilized to estimate the RFO, data symbols and channel state information (CSI) iteratively. Simulation results present that the proposed scheme outperforms the conventional RFO estimator [9] in all ranges of SNR and significantly compensates the performance degradation due to RFO.
The rest of this paper is organized in the following order. In section II, we describe the OFDM system to be considered. In section III, RFO compensation scheme based on the SAGE algorithm is proposed. Section IV presents the performance of the proposed algorithm over frequency selective fading channels evaluated by computer simulations. Some conclusion remarks are given in section $\mathrm{V}$.

\section{SYSTEM MODEL}

Let $N$ be the number of sub-carriers and $N_{s}=2 N_{\alpha}+1$ be the number of modulated sub-carriers. Note that $N-N_{s}$ sub-carriers at the edges of the spectrum are not used. Then, the timedomain received signal vector over frequency selective fading channel can be expressed as

$$
\mathbf{r}=\mathbf{F}_{v} \mathbf{F S D h}+\mathbf{w}
$$

where the normalized RFO $v$ is presented in the matrix $\mathbf{F}_{v}$ given by

$$
\mathbf{F}_{v}=\operatorname{diag}\left\{e^{j \frac{2 \pi v}{N} n}, 0 \leq n<N\right\}
$$

and the transmitted data symbols are given in the diagonal data symbol matrix

$$
\mathbf{S}=\operatorname{diag}\left\{s_{0}, s_{1}, \cdots, s_{N_{s}-1}\right\}
$$

The sub-carrier signals are shown in the matrix $\mathbf{F}$

$$
[\mathbf{F}]_{n, p}=\frac{1}{\sqrt{N}} \exp \left(j \frac{2 \pi\left(p-N_{\alpha}\right)}{N} n\right)
$$

where $n=0,1, \ldots, N-1$ and $\mathrm{p}=0,1, \ldots, N_{s}-1$. Moreover, the discrete Fourier transform (DFT) matrix $\mathbf{D}$ is given by

$$
[\mathbf{D}]_{p, l}=\exp \left(-j \frac{2 \pi\left(p-N_{\alpha}\right)}{N} l\right)
$$

where $l=0,1, \ldots, L-1$. In $(1), \mathbf{h}=[h(0) h(1) \cdots h(L-1)]^{T}$ and $\mathbf{w}=[w(0) w(1) \cdots w(N-1)]^{T}$ denote the channel impulse response (CIR) with $L$ multipaths and the additive white Gaussian noise vector, respectively. 


\section{PROPOSED SCHEME}

\section{A. RFO Compensation using SAGE Algorithm}

The frequency-domain received signal vector can be given as

$$
\begin{aligned}
\mathbf{z} & =\mathbf{F}^{H} \mathbf{r} \\
& =\varepsilon \cdot \mathbf{S D h}+\mathbf{R S D h}+\mathbf{v}
\end{aligned}
$$

where $(.)^{H}$ denotes the conjugated transpose. It is seen that $\varepsilon$ in (6) affects all sub-carriers constantly, which is defined by

$$
\varepsilon=\frac{1}{N} \sum_{n=0}^{N-1} e^{j \frac{2 \pi v}{N} n}
$$

In (7), assuming $v$ has a sufficiently small value and $\varepsilon$ is given, we can obtain $v$ approximately using Taylor's series expansion $\left(e^{j \frac{2 \pi v}{N} n} \approx 1+j \frac{2 \pi v}{N} n\right)$ shown as

$$
v \approx \frac{N}{\pi(N-1)} \operatorname{Im}\{\varepsilon\}
$$

Moreover, $\mathrm{R}$ in (6) induces the ICI due to RFO defined by

$$
\mathbf{R}=\mathbf{F}^{H} \mathbf{F}_{v} \mathbf{F}-\varepsilon \cdot \mathbf{I}
$$

where its diagonal terms are equal to zero.

In the proposed scheme based on SAGE algorithm, we intend to divide the received signal $\mathbf{z}$ in (6) into the desired signal

$$
\mathbf{z}_{D}=\varepsilon \cdot \mathbf{S D h}+\beta_{D} \mathbf{v}
$$

and the interference signal

$$
\mathbf{z}_{I}=\mathbf{R S D h}+\beta_{I} \mathbf{v}
$$

which indicates the transformation from the incomplete observation $\mathbf{z}$ to the complete observations $\mathbf{z}_{D}$ and $\mathbf{z}_{I}$ in the expectation-maximization (EM) algorithm [11]. Then, we try to estimate $\varepsilon, \mathbf{s}=\left[s_{0}, s_{1}, \cdots, s_{N_{s}-1}\right]^{T}$ and $\mathbf{h}$ using the desired signal $\mathbf{z}_{D}$ in (10).

In expectation step of the $(k)$ th iteration, the following expectations are evaluated

$$
\begin{aligned}
\mathbf{z}_{D}^{(k)} & =E\left[\mathbf{z}_{D} \mid \varepsilon^{(k)}, \mathbf{s}^{(k)}, \mathbf{h}^{(k)}\right] \\
& =\varepsilon^{(k)} \cdot \mathbf{S}^{(k)} \mathbf{D} \mathbf{h}^{(k)}+\beta_{D}^{2}\left(\mathbf{z}-\varepsilon^{(k)} \cdot \mathbf{S}^{(k)} \mathbf{D} \mathbf{h}^{(k)}-\mathbf{R}^{(k)} \mathbf{S}^{(k)} \mathbf{D} \mathbf{h}^{(k)}\right) \\
\mathbf{z}_{I}^{(k)} & =E\left[\mathbf{z}_{I} \mid \varepsilon^{(k)}, \mathbf{s}^{(k)}, \mathbf{h}^{(k)}\right] \\
& =\mathbf{R}^{(k)} \mathbf{S}^{(k)} \mathbf{D h}^{(k)}+\beta_{I}^{2}\left(\mathbf{z}-\varepsilon^{(k)} \cdot \mathbf{S}^{(k)} \mathbf{D h}^{(k)}-\mathbf{R}^{(k)} \mathbf{S}^{(k)} \mathbf{D h}^{(k)}\right)
\end{aligned}
$$

where $\varepsilon^{(k)}, \mathbf{s}^{(k)}$ and $\mathbf{h}^{(k)}$ denote the $(k)$ th estimates of $\varepsilon, \mathbf{s}$ and $\mathbf{h}$, respectively. Moreover, in (12) and (13), $\mathbf{R}^{(k)}=\mathbf{F}^{H} \mathbf{F}_{v^{(k)}} \mathbf{F}-\varepsilon^{(k)} \cdot \mathbf{I}$ where $v^{(k)} \approx N \operatorname{Im}\left\{\varepsilon^{(k)}\right\} / \pi(N-1)$. It is seen that the expectation step of the proposed algorithm is equivalent to the interference cancellation (IC) process in the received signals. Note that there is no need to evaluate $\mathbf{z}_{I}^{(k)}$ in (13), because the next maximization step employs only the desired signal $\mathbf{z}_{D}^{(k)}$ in (12).

The maximization step of the $(k)$ th iteration evaluates the following equations;

$$
\begin{gathered}
\varepsilon^{(k+1)}=\underset{\varepsilon}{\arg \max } \Lambda\left(\varepsilon, \mathbf{s}^{(k)}, \mathbf{h}^{(k)} \mid \mathbf{z}_{D}^{(k)}\right), \\
v^{(k+1)} \approx \frac{N}{\pi(N-1)} \operatorname{Im}\left\{\varepsilon^{(k+1)}\right\} \\
\mathbf{s}^{(k+1)}=\underset{\mathbf{s}}{\arg \max } \Lambda\left(\varepsilon^{(k+1)}, \mathbf{s}, \mathbf{h}^{(k)} \mid \mathbf{z}_{D}^{(k)}\right) \\
\mathbf{h}^{(k+1)}=\underset{\mathbf{h}}{\arg \max } \Lambda\left(\varepsilon^{(k+1)}, \mathbf{s}^{(k+1)}, \mathbf{h} \mid \mathbf{z}_{D}^{(k)}\right)
\end{gathered}
$$

where the log-likelihood function $\Lambda\left(\varepsilon, \mathbf{s}, \mathbf{h} \mid \mathbf{z}_{D}\right)$ can be given as

$$
\Lambda\left(\varepsilon, \mathbf{s}, \mathbf{h} \mid \mathbf{z}_{D}\right)=\operatorname{Re}\left\{\varepsilon \cdot \mathbf{z}_{D}^{H} \mathbf{S D h}\right\}-\frac{1}{2}|\varepsilon|^{2}|\mathbf{S D h}|^{2} .
$$

Then, the solution of (14) can be given as

$$
\varepsilon^{(k+1)}=\frac{1}{\left|\mathbf{S}^{(k)} \mathbf{D h}^{(k)}\right|^{2}}\left(\mathbf{S}^{(k)} \mathbf{D} \mathbf{h}^{(k)}\right)^{H} \mathbf{z}_{D}^{(k)}
$$

Moreover, assuming the constant envelope modulation and applying the least-square (LS) solution, (17) results in

$$
\mathbf{h}^{(k+1)}=\frac{\left(\varepsilon^{(k+1)}\right)^{*}}{\left|\varepsilon^{(k+1)}\right|^{2}} \mathbf{V}\left(\mathbf{S}^{(k+1)}\right)^{H} \mathbf{z}_{D}^{(k)}
$$

where $\mathbf{V}=\left(\mathbf{D}^{H} \mathbf{D}\right)^{-1} \mathbf{D}^{H}$. Note that it is known that $\beta_{D}=\beta_{I}=1$ is the optimal condition to maximize the Fisher information of $\mathbf{z}_{D}$ and $\mathbf{z}_{I}[10]$.

\section{B. Initialization}

The initial CSI $\mathbf{h}^{(0)}$ can be given by the channel estimate in previous OFDM symbol assuming that the channel variation between adjacent OFDM symbols is negligible. For $\varepsilon^{(0)}$ and $\mathbf{s}^{(0)}$, however, the initial estimates should be evaluated by the received signal including ICI, because IC process can not be operated without the information of $s$.

In order to obtain $\varepsilon^{(0)}$, we use pilot sub-carriers embedded in each OFDM symbol shown as

$$
\varepsilon^{(0)}=\frac{1}{\left|\mathbf{S}_{p} \mathbf{D}_{p} \mathbf{h}^{(0)}\right|^{2}}\left(\mathbf{S}_{p} \mathbf{D}_{p} \mathbf{h}^{(0)}\right)^{H} \mathbf{z}_{p}
$$

where $\mathbf{z}_{p}, \mathbf{S}_{p}$ and $\mathbf{D}_{p}$ denote the received signal vector at the pilot sub-carriers, the diagonal pilot symbol matrix and the DFT matrix composed of only pilot sub-carriers, respectively. Moreover, given $\varepsilon^{(0)}$, we can obtain $v^{(0)}$ as shown in (8). For $\mathbf{s}^{(0)}$, we evaluate the following equation 


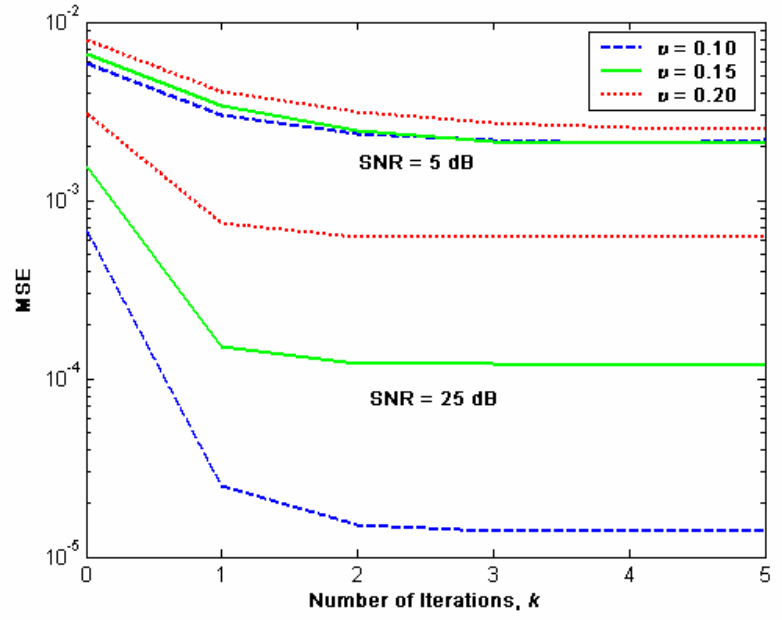

(a)

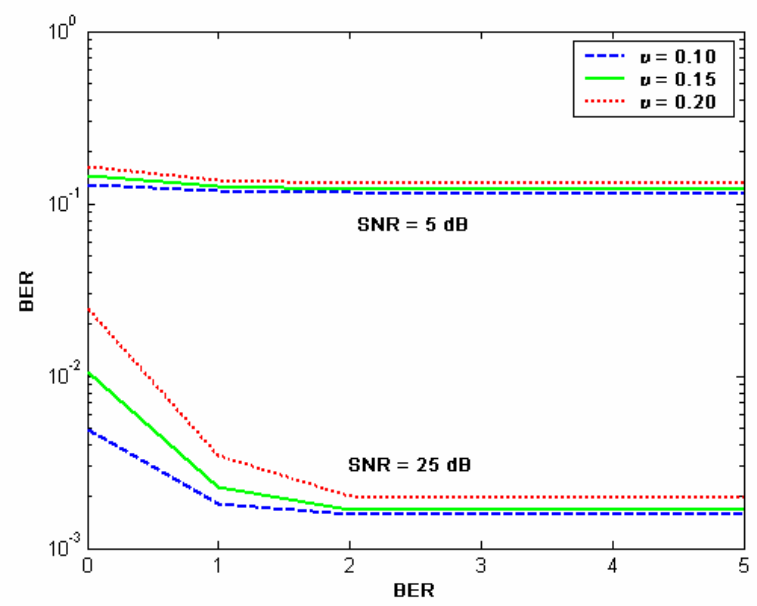

(b)

Figure 1. Convergence properties of proposed algorithm. (a) MSE performance of the normalize RFO $v$, (b) BER performance.

$$
\mathbf{s}^{(0)}=\underset{\mathbf{s}}{\arg \max } \Lambda\left(\varepsilon^{(0)}, \mathbf{s}, \mathbf{h}^{(0)} \mid \mathbf{z}\right)
$$

Note that $\varepsilon^{(0)}$ and $\mathbf{s}^{(0)}$ are very coarse estimates because $\mathbf{z}_{p}$ and $\mathbf{z}$ in (21) and (22) fully contain the ICI due to RFO.

\section{Simulation Results}

System parameters for our simulations follow IEEE 802.11a [12] and the sub-carriers are modulated by quadrature phase shift keying (QPSK). The FFT size and the number of modulated sub-carriers are given by $N=64$ and $N_{s}=53$, respectively. It is assumed that the power delay profile of CIR is given as $|h(l)|^{2} \propto e^{-l / 4}, 0 \leq l<8$ and each multipath varies according to Rayleigh distribution [13]. Moreover, one packet is composed of 100 OFDM symbols and the first symbol of each packet is set to be a preamble. It is assumed that the RFO is constant in each packet and the process for RFO in (14) and (15) is performed only at the first OFDM data symbol after the

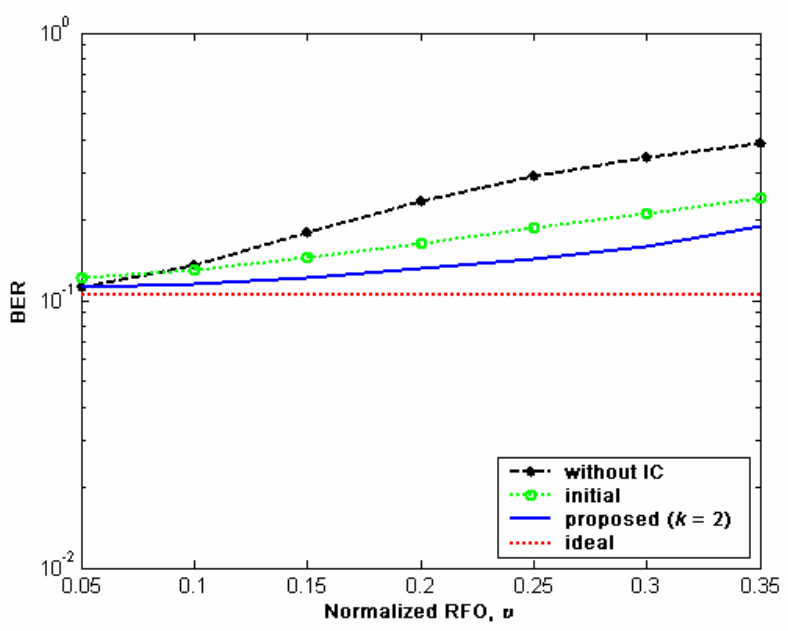

(a)

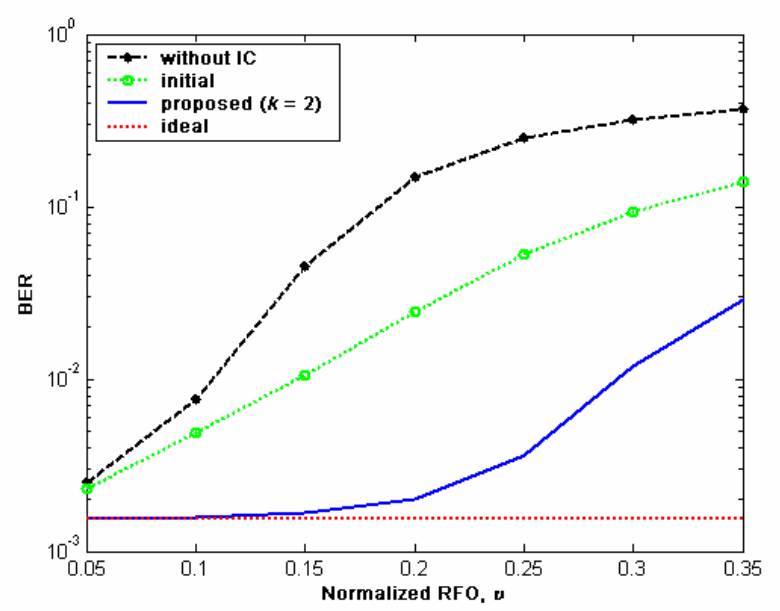

(b)

Figure 2. Comparisons of BER performance according to the different values of normalized RFO $v$. (a) $\mathrm{SNR}=5 \mathrm{~dB}$, (b) $\mathrm{SNR}=25 \mathrm{~dB}$.

preamble. The final estimate of RFO is utilized at the other OFDM data symbols in the packet.

At first, we consider a static channel, which indicates that the channel is constant over a packet and the CSI is available at the receiver. Then, there is no need to include the process for CSI in (17). Fig. 1 shows the convergence properties of the proposed algorithm. It is seen that two iterations are enough to obtain the converged mean square error (MSE) performance of RFO estimate and bit error rate (BER) performance. Therefore, we fix the number of iterations to two in the following simulation results.

In Fig. 2, we present the BER performance of proposed algorithm according to the different values of the normalized RFO $v$. The performance is also compared with ideal performance without RFO, performance with no IC and those of initialization process described in section 3.2. It can be concluded that the proposed algorithm significantly compensates the performance degradation especially in high signal to noise ratio (SNR) and it achieves almost ideal 


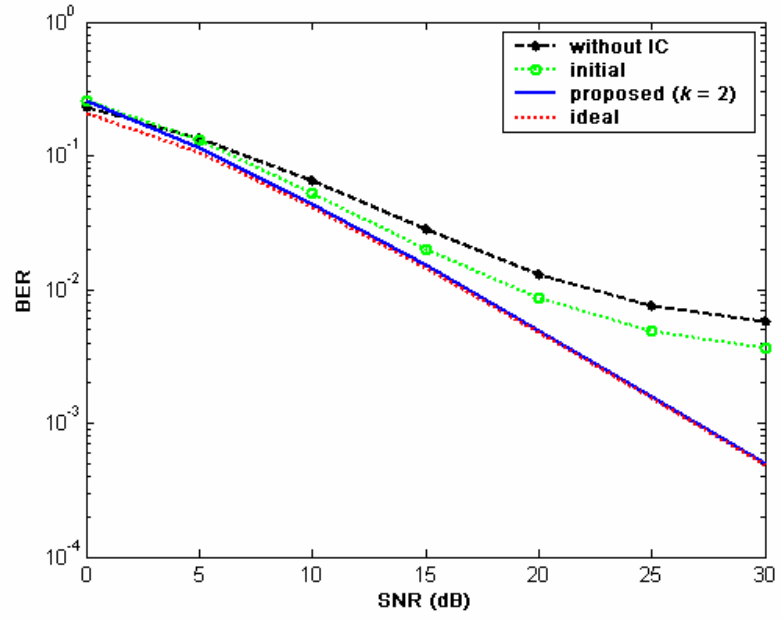

(a)

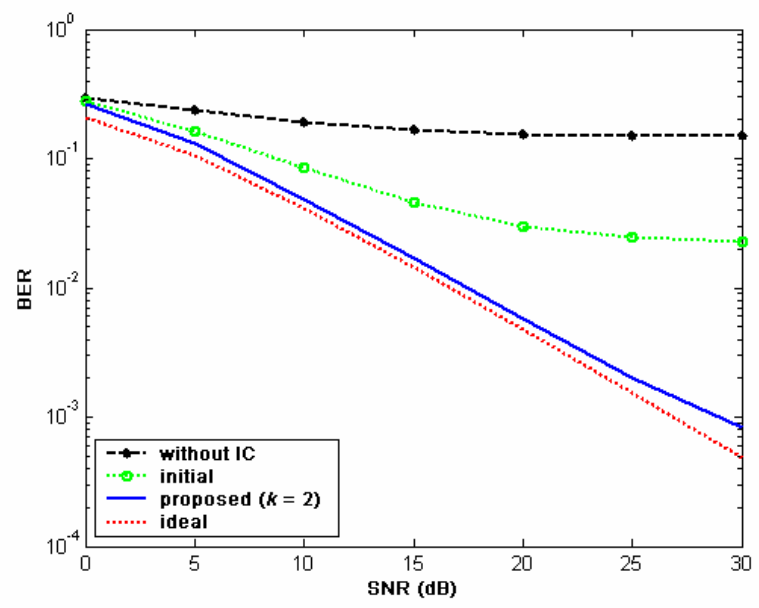

(b)

Figure 3. BER versus SNR in static-channels. (a) the normalize RFO $v=$ 0.1 , (b) the normalize RFO $v=0.2$.

performance as long as $v<0.2$. Fig. 3 shows the BER performance versus SNR. It can be also observed that, in all ranges of SNR, the proposed scheme achieves ideal performance and remarkably compensates the performance degradation due to RFO.

The following results show the BER performance of the proposed algorithm for a time-varying channel, which indicates that the channel variation should be tracked at each OFDM symbol. In our simulation, it is assumed that the carrier frequency and vehicle speed are set to be $5 \mathrm{GHz}$ and $60 \mathrm{Km} / \mathrm{h}$, respectively. In this case, the proposed algorithm should include the CSI process in (17).

Fig. 4 presents the MSE performance of proposed RFO estimator in (15) compared with that of conventional schemes in [3] and [9] over time-varying multipath channels. In our simulations, [3] uses the cyclic prefix composed of 16 samples and [9] employs 10 sub-carriers that are not actually modulated and the truncation factor of Taylor's series is assumed to be one. It is seen that the proposed RFO estimator outperforms the

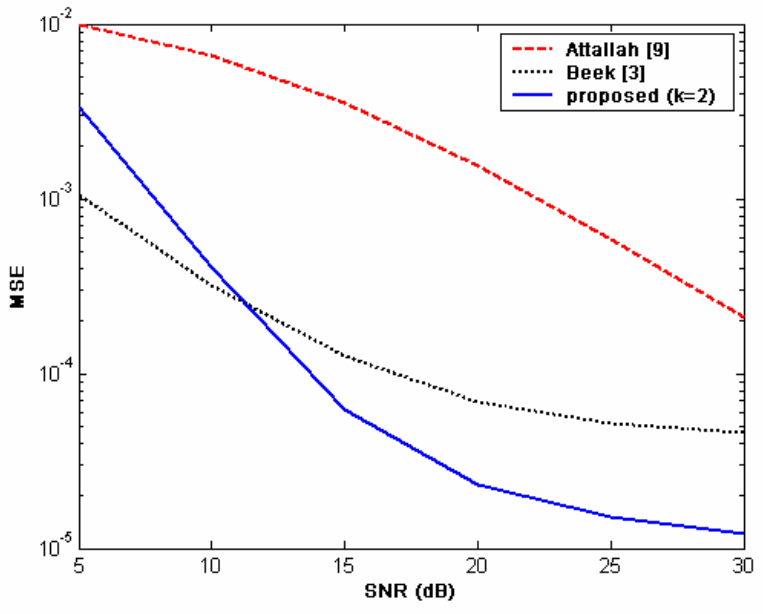

Figure 4. Comparisons of MSE performance versus SNR for RFO estimator in time-varying channels at the normalize RFO $v=0.1$.

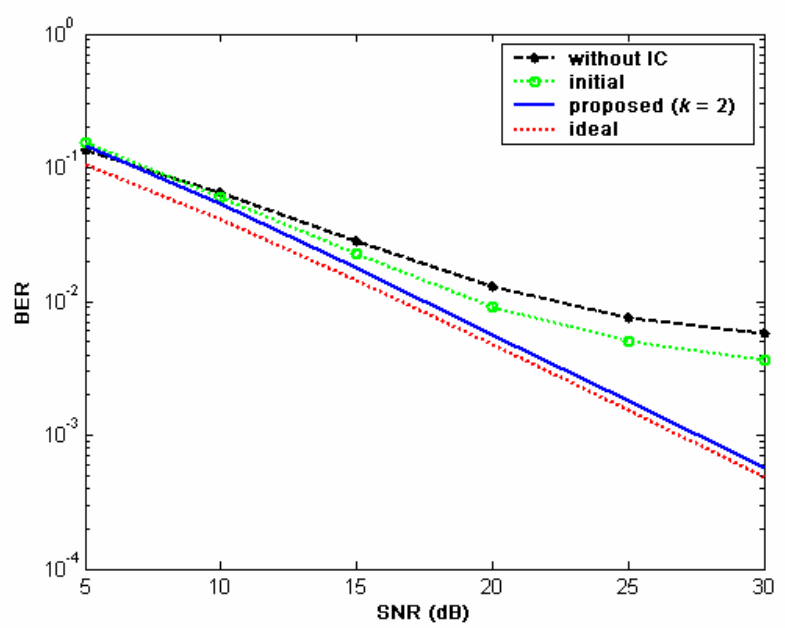

(a)

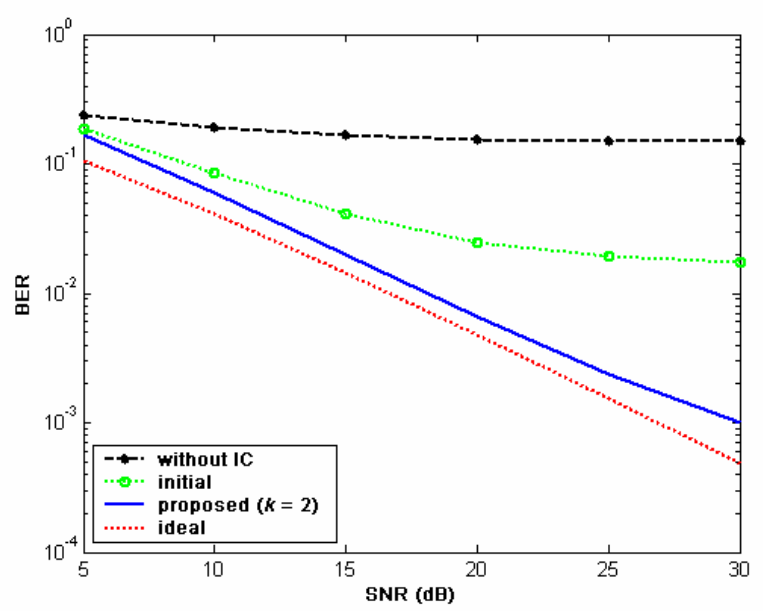

(b)

Figure 5. BER versus SNR in time-varying channels. (a) the normalize RFO $v=0.1$, (b) the normalize RFO $v=0.2$. 
conventional scheme in [9]. Moreover, in high SNR, the proposed scheme shows performance advantage compared with [3]. Fig. 5 indicates that the degraded performance due to RFO is also compensated significantly by the proposed algorithm in time-varying channels.

\section{CONCLUSION}

In this paper, we propose an iterative detection scheme using the SAGE algorithm in the presence of RFO for OFDM system. The expectation step in the proposed scheme extracts the desired terms from the received signals. Then, the maximization step is utilized to estimate the RFO, data symbols and CSI using the desired signals. Simulation results show that the proposed scheme needs two iterations to obtain the converged estimates. Moreover, it significantly compensates the performance degradation due to RFO and achieves almost ideal performance in all ranges of SNR, while the normalized RFO value is within 0.2 .

\section{ACKNOWLEDGMENT}

This work was supported by University IT Research Center Project.

\section{REFERENCES}

[1] M. Speth, S. A. Fechtel, G. Fock, and H. Meyr, "Optimum receiver design for wireless broad-band systems using OFDM-part I," IEEE Trans. Commun., vol. 47, no. 11, pp. 1668-1677, Nov. 1999.

[2] F. Daffara, and O. Adami, "A new frequency detector for orthogonal multicarrier transmission techniques," in Proc. Vehic. Technol. Conf, vol. 2, July 1995, pp. 804-809.
[3] J. J. van de Beek, M. Sandell, and P. O. Borjesson, "ML estimation of timing and frequency offset in OFDM systems," IEEE Trans. Signal. Processing, vol. 45, no. 7, pp. 1800-1805, Jul. 1997.

[4] T. M. Schmidl, and D. C. Cox, "Robust frequency and timing synchronization for OFDM," IEEE Trans. Commun., vol. 45, no. 12, pp. 1613-1621, Dec. 1997.

[5] A. J. Coulson, "Maximum likelihood synchronization for OFDM using a pilot symbol : algorithm," IEEE J. Select. Areas Commun., vol. 19, pp. 2486-2494, Dec. 2001.

[6] F. Tufvesson, O. Edfors, and M. Faulkner, "Time and frequency synchronization for OFDM using PN-sequence preamble," in Proc. Vehic. Technol. Conf, vol. 4, Sept. 1999, pp. 2203-2207.

[7] X. Wang, T. T. Tjhung, Y. Wu, and B. Caron, 'SER performance evaluation and optimization of OFDM system with residual frequency and timing offsets form imperfect synchronization," IEEE Trans. Broadcast, vol. 49, no. 2, Jun. 2003, pp. 170-177.

[8] H. Lui, and U. Tureli, "A high efficient carrier estimator for OFDM communications," IEEE Commun.Lett., vol. 2, no. 4, pp. 104 106, Apr. 1998.

[9] S. Attallah, "Blind estimation of residual carrier offset in OFDM systems," IEEE Signal Processing Lett., vol. 11, no. 2, pp. 216 219, Feb. 2004 .

[10] J. A. Fessler, and A. O. Hero, "Space-alternating generalized expectation-maximization algorithm," IEEE Trans. Signal Processin., vol. 42, no. 10 , pp. 2664 2677, Oct. 1994.

[11] C. N. Georghiades, and J. C. Han, "Sequence estimation in the presence of random parameters via the EM algorithm," IEEE Trans. Commun., vol. 45, no. 3, pp. 300-308, Mar. 1997.

[12] IEEE 802.11 WG, part 11: Wireless LAN Medium Access Control (MAC) and Physical Layer (PHY) Specifications : High-speed Physical Layer in the $5 \mathrm{GHz}$ band, Supplement to IEEE 802.11 Standard, Sep. 1999.

[13] W. C. Y. Lee, Mobile Communications Engineering, New-York: McGrow-Hill, 1982. 